\title{
Plant Stress Hormone Methyl Jasmonate: Potential Candidate for Human Therapeutic Applications
}

\author{
Gunjegaonkar SM${ }^{1}{ }^{*}$, Shanmugarajan TS${ }^{1}$ and Wankhede SB ${ }^{2}$ \\ ${ }^{1}$ Vels Institute of Science, Technology and Advanced Studies, India \\ 2Professor and Principal, Department of Pharmaceutical Chemistry, JSPM's Charak \\ College of Pharmacy and research, India
}

Editorial

Volume 2 Issue 3

Received Date: May 30, 2018

Published Date: June 06, 2018

*Corresponding author: Gunjegaonkar Shivshankar, School of Pharmaceutical Sciences, Vels Institute of Science, Technology and Advanced Studies, Chennai - 600 117, India, Tel: +91-9326006825; Email: gunjeshiv@gmail.com

\section{Editorial}

The chemical substances synthesized by the plants which regulated the overall growth and development are called as "Phytohormones" or "Plant hormones" sometimes referred as "plant growth substances". Similar to human hormones they are synthesized by specialized cells in a very low concentration and regulate cellular processes in targeted cells locally or migrate to target cells. Phytohormones are important for the formation of leaves, stems, flowers, fruits, and also shaping and deciding the size of the plants. In 1937 went and thimann addresses these chemical substances as 'Phytohormone'.

The important phytohormones like Abscisic acid, Auxins, Brassinosteroids, Cytokinins, Ethylene, Gibberellins, Jasmonates, and Salicylic acid Strigolactones have been identified with their potential role in the plant. Among them, Abscisic acid, ethylene, Jasmonic acid, and salicylates are the most common Plant Stress Hormones (PSH) and are screened for their beneficial potential to human beings. A salicylic acid derivative like aspirin has expensively studied for its therapeutic application. In plants, these stress hormones play an important role to activate the cellular responses for diverse stress conditions like wear and tear injuries or environmental stresses, such as ultraviolet radiation, osmotic shock, and heat etc. Today scientists are keenly interested to explore the potential benefits of jasmonate and its derivatives. The jasmonates are the lipid-based hormones that were originally isolated from jasmine oil. The most active Jasmonate in plants is jasmonic acid. Methyl jasmonate is a metabolized derivative of Jasmonic acid [1].
In the traditional medicinal system, the Chameli plant/ Spanish Jasmine (Asminum Officinale Linn.) contains an abundant amount of jasmonic acid derivatives accumulated in the flowers [2]. Ayurvedic literature advocated that oil obtained from jasmine flower have a calming and sedative effect, Nervous system depressant, mild anesthetic and astringent property. The flowers and plant parts are used as a diuretic, cough suppressant, anthelmintic, ulcer curative, and in certain skin diseases. Methyl Jasmonate is highly concentrated in flowers and leaf and used for treating a tumor [3].

Recent studies scientifically revealed that methyl jasmonate is successfully able to control the cancerous cell development and proves its potential anticancer activity in different types of cancers namely Prostate cancer, Skin cancer, Liver cancer, Bladder cancer, Neuroblastoma, oral cancer, Lung cancer, Endometrial cancer, cervical cancer, colon cancer etc. Various underline mechanisms have been proposed for its anticancer action. The first mechanism is inhibition of Lipo-oxygenase (LOX-5) an important mediator in the cancerous cell. Secondly, Suppression of anti-apoptotic protein causes arrests of cell cycle and inhibition of cell growth and proliferation. The third proposed mechanism is ATP depletion in cancer cells via mitochondrial perturbation, a detachment of hexokinase from the mitochondria, induction of apoptosis in cancerous cells, decreases' expression of cancerous cell regulator etc [4].

20 methyl jasmonate derivatives were synthesized and screened for its anti-inflammatory activity. Four $\alpha$ - 
haloenonejasmonates derivatives exhibit significant antiinflammatory activity in LPS stimulated RAW cells. The effect is contributed by inhibiting pro-inflammatory mediators (NO, IL-6, and TNF- $\alpha$ ) [5]. Anti-inflammatory and membrane stabilizing properties of methyl jasmonate was evaluated in rats and showed promising potential. The anti-inflammatory effect is correlated with Methyl jasmonate prominent anti-oxidant activity [6]. Some investigation revealed that methyl jasmonate reduced nociceptive responses associated with inflammatory conditions in animals and provides experimental evidence that may support its potential applications in the treatment of inflammatory pain [7]. Methyl jasmonate was successfully screened for antimalarial activity in experimental animals. The effect was confirmed by remarkable reduction in percentage parasitemia [8]. Methyl jasmonate is acting as an anti-stress hormone in the plant considering the same principles scientist investigates antianxiety effect in experimental animals. The stress is an important factor in the generation of anxiety. This study reveals that Methyl jasmonate exhibits an adaptogenic property in providing resistance against stressful conditions in animals [9].

Methyl Jasmonate significantly enhances memory performance and experimentally induced cognitive impairment in mice. This study reveals that the underline mechanism was significant inhibition of acetylcholinesterase thereby increases cholinergic transmission in the brain, in addition, significant antioxidant activity in the brain cells.

In the conclusion, Methyl jasmonate is not only important for plants overall growth, development, and survival to stressful conditions but also stands as a future important candidature for the human being. The various studies have been carried out using experimental animals and isolated cell lines but not in the human being moreover studies are limiting the exact molecular mechanism behind existing effect of methyl jasmonate.

As a process of drug discovery and development Methyl jasmonate is effective in experimental animals with minimum know toxicities further investigation are needed in the human being to bring $\mathrm{MJ}$ as a potent drug for treatment of various disease and disorder.

\section{References}

1. Sembdner G, Parthier B (1993) The biochemistry and the physiological and molecular actions of jasmonates. Annu Rev Plant Physiol Plant Mol Biol 44: 569-589.

2. Buchbauer G (1990) Do essential oils have therapeutic properties? Perfumer \& flavorist Aroma therapy 15: 47- 50.

3. Khare CP (2007) Indian medicinal plants an illustrated dictionary. $1^{\text {st }}$ (Edn.), Springer-Verlag New York, pp: 343-344.

4. Gunjegaonkar SM, Shanmugarajan TS (2016) Methyl jasmonate: New insights into a potent phytohormone. Int J Pharm Bio 7(1): 244-249.

5. Dang H, Lee H, Yoo ES, Hong J, Bao B, et al. (2008) New jasmonate analogues as potential anti-inflammatory agents. Bioorganic \& Medicinal Chemistry 16(24): 10228-10235.

6. Solomon U, Akinyinka A (2017) Anti-inflammatory and membrane stabilizing properties ofmethyl jasmonate in rats. Chinese Journal of Natural Medicines 15(3): 0202-0209.

7. Abimbola S, Olugbemide, Umukoro S (2011) Antinociceptive effects of methyl jasmonate in experimental animals. J Nat Med 65(3-4): 466-470.

8. Oladapo EO, Ayokulehin MK (2015) Potential antimalarial activity of Methyl Jasmonate and its effect on lipid profiles in Plasmodium Berghei infected mice. African Health Sciences 15 (3): 841-850.

9. Oritoke MA, Solomon U (2015) Effects of Methyl Jasmonate on Acute Stress Responses in Mice Subjected to Forced Swim and Anoxic Tests. Sci Pharm 83(4): 635-644. 